\title{
Could docosahexaenoic acid (DHA), found in breast milk and artificial baby milks, protect infants and children from COVID-19 and make them less susceptible to COVID-19-related severe illness, as well as treat adult COVID-19 patients in combination with retinoic acid? A plan for observation and intervention
}

Mahmoud Ramadan Elkazzaz ( $\square$ mahmoudramadan2051@yahoo.com )

Department of chemistry and biochemistry, Faculty of Science, Damietta University, Egypt https://orcid.org/0000-0003-3703-520X

Amr kamel khalil Ahmed ( $\nabla$ drmedahmed@gmail.com )

Director of tuberculosis program Ghubera, public health department ,First health cluster ,Ministry of health, Riyadh, Saudia Arabia https://orcid.org/0000-0003-3477-236X

Method Article

Keywords: COVID-19, Children, Infants, Adults, DHA, Milk Formula, Retinoic Acid

Posted Date: October 11th, 2021

DOl: https://doi.org/10.21203/rs.3.rs-960227/v1

License: (c) (i) This work is licensed under a Creative Commons Attribution 4.0 International License.

Read Full License 


\section{Abstract}

SARS-CoV-2, which causes COVID-19, is a new virus that has spread fast over the world. The severity of COVID-19 at different ages has been a notable and constant observation: severity, the requirement for hospitalization, and mortality all grow sharply with age, although severe disease and death are uncommon in children and young adults.. The majority of children infected with SARS-CoV-2 are asymptomatic or have moderate symptoms, which include fever, cough, pharyngitis, gastrointestinal symptoms, and changes in taste and smell. The question of whether children are less likely to be infected with SARS-CoV- 2 is still being debated. Children make up only 1 to $2 \%$ of all SARS-CoV- 2 cases, according to large epidemiological research. these numbers are heavily, depend on testing criteria, and in many reports, testing was limited to those who were symptomatic or required hospitalization, which is less common in children.. According to certain research, children are just as likely as adults to contract SARSCoV-2.9. Recent research suggests that children are less likely to become infected after coming into touch with a SARS-CoV-2-positive person.According to some reports, children and adolescents have similar virus loads and are hence just as likely to transmit SARS-CoV-2 as adults. Furthermore, the viral load in asymptomatic and symptomatic people may be identical. Reassuringly, transmission of the virus from children to other children or adults in schools has been infrequent.Children are less likely to be infected with SARS-CoV-2 and have less severe symptoms, which is similar to what has been observed with SARSCoV-1 and Middle East respiratory disease (MERS)-CoV. Infection with most other respiratory viruses (e.g., respiratory syncytial virus (RSV), metapneumovirus, parainfluenza, or influenza viruses), on the other hand, has a far higher prevalence and severity in youngsters. Dr. Mahmoud Elkazzaz and Dr Amr kamel khalil Ahmed, the lead investigators of this observational study, recently published a preprint that demonstrated Docosahexaenoic acid (DHA) had a high binding affinity and greatest interactions with ACE2 active sites, as well as a moderate binding affinity and moderate interactions with the active sites of IL-6. The Docosahexaenoic acid (DHA) interacts with different active sites of IL6 and ACE2 which are involved in direct or indirect contacts with the ACE2 and IL- 6 receptors which might act as potential blockers of functional ACE2 and IL- 6 receptor complex.. A study proposed, a clinical benefit of targeting IL-17A signaling and the synergic inflammatory cytokine IL- 6 to manage COVID-19 patients, particularly those presenting with cytokine storm syndrome.Hypercytokinemia, caused by notably high proinflammatory cytokines such as interleukin (IL)-1B, IL-6, IL-8, and IL-17, is mostly linked to the worsened clinical presentation of COVID-19 patients(14). In PBMCs from individuals with relapsing-remitting multiple sclerosis, a combination of docosahexaenoic acid (DHA) and all-trans-retinoic acid (ATRA) inhibits IL-17 gene expression.

\section{Conclusions}

Docosahexaenoic acid (DHA) was detected in abundance in breast milk and other algal sources milk supplement used for newborns and children's feeding. As a result, we believe that docosahexaenoic acid (DHA) may protect children and newborns thorough competing with COVID-19 for ACE2 receptors and inhibiting IL-6 activity and may possibly help them avoid a cytokine storm and save their lives through inhibiting IL-6 and preventing SARS- CoV-2 RBD attachment to ACE2. In addition to IL-17 was fond to 
increase COVID-19 inflammatory complication in this case DHA combined with retinoic acid is expected to be effective in inhibiting IL-6 and IL-17.

\section{Introduction}

The COVID-19 pandemic caused by severe acute respiratory syndrome coronavirus 2 (SARS-CoV-2) has infected over 235 million people causing over 4.8 million deaths over the world, and it is still expanding especially since current vaccines have not been able to completely end the pandemic because of existing new variants(1).

The world is still in an urgent need for targeted and effective COVID-19 treatments which has put great pressure on researchers across the world for developing effective drugs. For severe acute respiratory syndrome coronavirus 2, the lower airway is the major target of infection (SARS-CoV-2). Pneumonia is almost always prevalent in COVID-19 patients(2,3). COVID-19 appears to be uncommon in youngsters, according to available reports $(4,5,6,7)$. According to recent data from the Chinese Centers for Disease Control and Prevention, $416(0.9 \%)$ of the 44,672 confirmed COVID-19 cases as of February 11, 2020 were aged $0-10$ years and $549(1.2 \%)$ were aged $10-19$ years(8). Investigating the fundamental causes may aid in the understanding of COVID-19 pathophysiology.

Based on our recently published preprint , in which we found that Docosahexaenoic acid (DHA), a substance found abundantly in breast milk and added to other artificial milk formula had a high binding affinity and greatest interactions with ACE2 active sites, as well as a moderate binding affinity and moderate interactions with the active sites of IL-6(16). The Docosahexaenoic acid (DHA) interacts with different active sites of IL6 and ACE2 which are involved in direct or indirect contacts with the ACE2 and IL-6 receptors which might act as potential blockers of functional ACE2 and IL-6 receptor complex(16) as showed in table 2 .

DHA is found in breast milk, the mean (+/-SD) concentration of DHA in breast milk (by wt) is $0.32+/-$ $0.22 \%$ (range: $0.06-1.4 \%$ ) and that of AA is $0.47+/-0.13 \%$ (range: $0.24-1.0 \%$ )(9). Docosahexaenoic acid (DHA) is found in breast milk and has a crucial function in the development of young children.

Endogenous synthesis is insufficient to maintain tissue levels equal to breast-fed infants, hence a supply of these fatty acids in infant formula (usually following breast milk as a model with ARA > DHA) is regarded to be important. Numerous good developmental results (closer to breast-fed infants) have been found in intervention trials evaluating the influence of DHA- and ARA-supplemented formulas, including measurements of specific cognition functions, visual acuity, and immunological responses (10). In vitro and animal experiments, DHA has been shown to reduce the release of inflammatory cytokines(11). Calu3 cells infected with Rhinovirus RV-43 and RV-1B released considerably less IL- 6 and IP-10 after being supplemented with DHA (400 mM)(12).

High-dose (1.5 g/day EPA and $1.0 \mathrm{~g} /$ day DHA) n-3 supplementation can lower plasma levels of both IL-6 and IL-1ß, according to the findings of a randomised, controlled trial published in 2018(12). 
Naturally, cow's milk does not provide a rich source of DHA however in North America whole milk and partially skimmed milk (2\%) are fortified with DHA by adding DHA rich feed additive to cattle's diet. Skim milk or low fat milk (1\%) cannot be fortified with DHA because DHA is contained in the milk fat(13).

Supplementing with EPA and DHA appears to have an anti-inflammatory impact, which is consistent with the majority of previous clinical findings as in table 1 (12).

A study proposed, a clinical benefit of targeting IL-17A signaling and the synergic inflammatory cytokine IL-6 to manage COVID-19 patients, particularly those presenting with cytokine storm syndrome(14).Hypercytokinemia, caused by notably high pro-inflammatory cytokines such as interleukin (IL)-1B, IL-6, IL-8, and IL-17, is mostly linked to the worsened clinical presentation of COVID-19 patients(14). In PBMCs from individuals with relapsing-remitting multiple sclerosis, a combination of docosahexaenoic acid (DHA) and all-trans-retinoic acid (ATRA) inhibits IL-17 and RORt gene expression(15).

Table 1

Table 2. Docosahexaenoic acid (DHA) binding affinities with ACE2 and IL-6 in addition to its other therapeutic properties 
Docosahexaenoic acid (DHA) binding affinities with ACE2 and IL-6 could make it a potent protective factor against COVID-19 in children and Infants
Binding Affinity
$-6.3$
with ACE2
receptor
Binding Affinity $\quad-5.3$
with IL-6

Hydrogen bonds Two strong hydrogen bonds

with ACE2

Hydrogen bonds One strong hydrogen bond

with

IL-6

Type Omega-3 fatty acid

Anti-inflammatory Inhibition of Interleukin-6(IL-6) (Rehman K et al.,2016)

properties

Inhibition of tumor necrosis factor-alpha (TNF-a) and nuclear factor-KB (NF-KB)

(Yang YC et al.,2013)

Smell, taste and Protective for the olfactory system ( Gao MG et al.,2021)

GPCRs

DHA levels affect ad modulates $G$ protein-coupled receptors (GPCRs)

function (Guixà-González R et al., 2016)

Sources

Breast Milk, Fish oils, Single cell oils (SCO) (Algal source like Crypthecodinium and Schizochytrium)( Z.Cohen et al,. 2010)

Anti-clotting

properties

Inhibition of platelet aggregation (DiNicolantonio JJ et al.,2019)

DHA for children DHA has many health benefits for both mother and baby (Juber et al,.2016)

1. Enhances brain function and vision. (Horrocks LA.,1999)

2. Treats allergies, asthma, and attention deficit-hyperactivity disorder (ADHD). (Miyata J et al., 2015)

DHA , COVID-19 and

Children
According to our findings DHA found in breast milk (Juber et al,.2016), could act as a protective factor against COVID-19 via inhibiting both

ACE2 receptors and IL-6

\section{Patients And Methods}


Groups/Cohorts

300 Newborn feeding on Breast Milk or Artificial Milk Formula from birth to

about 2 months of age

The electronic patient record of 300 Newborn feeding on Breast Milk or Artificial Milk Formula from birth to about 2 months of age would be examined and followed between March 2020 and October 2021 and compare their rates of Covid-19 infection, hospitalization and complications with the rates of the Control Groups

300 Infant feeding on Breast Milk or Artificial Milk Formula from 2 months to

1 year

The electronic patient record of 300 Infant feeding on Breast Milk or Artificial Milk Formula from 2 months to 1 year would be examined and followed between March 2020 and October 2021 and compare their rates of Covid-19 infection, hospitalization and complications with the rates of the rates of the Control Groups

300 child feeding on Breast Milk or Artificial Milk Formula from 1 year to 5

year

The electronic patient record of 300 child feeding on Breast Milk or Artificial Milk Formula from 1 year to 5 year would be examined and followed between March 2020 and October 2021 and compare their rates of Covid-19 infection, hospitalization and complications with the rates of the rates of the Control Groups

300 child feeding on Breast Milk or Artificial Milk Formula from 5 year up to

15 year

The electronic patient record of 300 child feeding on Artificial Milk Formula or any source of DHA from 5 year up to 15 year would be examined and followed between March 2020 and October 2021 and compare their rates of Covid-19 infection, hospitalization and complications with the rates of the Control Groups

300 adult patients from 15 years to 25 years infected with COVID-19 will be treated by taking Milk formula supplement or capsules

contain DHA for one month

300 adult patients from 15 years to 25 years infected with COVID-19 will be treated by taking Milk formula supplement or capsules

contain DHA and retinoic acid capsules(Tretinoin 10mg soft capsules) for one month

Control Group

1. The electronic patient record of 150 infant and child patients who feed on only cow's milk which does not provide a rich source of

DHA and did not feed on any Artificial Milk Formula or any source of DHA would be examined and followed between March 2020 and October 2021 and compare their rates of Covid-19 infection, hospitalization and complications with the rates of the cohorts that feed on Breast Milk or Artificial Milk Formula

2. The electronic patient record of 150 adult patients from 15 years to 25 years who did not feed on any Artificial Milk Formula or any source of DHA would be examined and followed between March 2020 and October 2021 


\section{Anticipated Results and Outcome Measures of children and infants}

Primary Outcome Measure:

. Susceptibility for COVID-19.

Did infants and children who were fed breast milk or artificial milk formula have a lower risk of infection with Covid-19? in comparison with the control groups

[Time Frame: March2020 - October 2021]

Secondary Outcome Measure:

Severity for COVID-19.

Did infants and children with COVID-19 who were fed breast milk or artificial milk formula have a lower chance of illness severity and requiring intensive care support or not in comparison with the control groups, ?

[Time Frame: March2020 - October 2021]

Mortality

In comparison to the control groups, did newborns and children with COVID-19 who were fed breast milk or artificial milk formula have a reduced mortality rate?

[Time Frame: March2020 - October 2021]

Care and Critical Care

Did infants and children with COVID-19 feeding on Breast Milk or Artificial Milk Formula have a shorter Length of hospitalization and stay in the ICU in comparison with the control groups

[Time Frame: March2020 - October 2021]

Resolution of fever

In comparison to the control groups, did COVID-19-infected infants and children who were fed breast milk or artificial milk formula have a faster resolution of fever and did not require advanced fever management?

[Time Frame: March2020 - October 2021]

Percentage of children requiring intensive 
care support [Time Frame: March2020 - October 2021]

Clinical symptoms of children

[Time Frame: March2020 - October 2021]

\section{Anticipated Results Outcome Measures of Adults}

1.lung injury score

Proportion of lung injury score decreased or increased after treatment

[Time Frame: at 7 and 14 days]

Secondary Outcome Measures:

Absolute lymphocyte counts (CD4,CD8 and CD25+FOXP3+ Regulatory T)

[Time Frame: at day 7 and 14 after randimization]

Serum levels of IL-6,TNF,TLR3,CRP, ESR and Type I interferon

[Time Frame: at day 7 and 14 after randimization]

Serum level of COVID19 RNA

Serum level of COVID19 RNA

[Time Frame: at day 7 and 14]

All-cause mortality rate

died

[Time Frame: at day 7 and 14]

Ventilation free days

ventilation free days

[Time Frame: at 14 days]

ICU free days

ICU free days 
[Time Frame: at 14 days]

d-dimers

less than $250 \mathrm{ng} / \mathrm{mL}$, or less than $0.4 \mathrm{mcg} / \mathrm{mL}$ of blood sample

[Time Frame: at 3-5days]

Time to first negative SARS-CoV-2 PCR in NP swap

(if pos. at baseline)

[Time Frame: within 14 days]

Angiotensin 1-7 (Ang 1-7) changes over time

[Time Frame: at day 7 and 14]

Angiotensin 1-5 (Ang 1-5) changes over time

[Time Frame: at day 7 and 14]

Renin changes over time

[Time Frame: at day 7 and 14]

Aldosterone changes over time

[Time Frame: at day 7 and 14]

Angiotensin-converting enzyme II (ACE2) changes over time

[Time Frame: at day 7 and 14]

Frequency of adverse events and severe adverse events

[Time Frame: 14 days]

Angiotensin II (Ang II) changes over time

[Time Frame: at day 7 and 14]

Sequential organ failure assessment score(SOFA score) over time

[Time Frame: at day 7 and 14]

Transe membrane protease, serine II (TMPRSS2) changes over time 
[Time Frame: at day 7 and 14]

Testosterone levels changes over time

[Time Frame: at day 7 and 14]

Dihydrotestosterone(DHT) levels changes over time

[Time Frame: at day 7 and 14]

Cholesterol levels changes over time

[Time Frame: at day 7 and 14]

Thrombin time (TT)

[Time Frame: at day 7 and 14]

$\lg A$ antibodies

[Time Frame: at day 7 and 14]

Melanoma differentiation-associated protein-5, retinoic acid inducible gene-1 (RIG-1)

[Time Frame: at day 7 and 14]

\section{Drug used}

1-Tretinoin $10 \mathrm{mg}$ soft capsules

2-DHA supplement

3-DHA in Milk formula

\section{Study Description}

- The study is configured as a retrospective and prospective observational in children and infants and interventional in adults study. The study will be multi-center and will involve all Infants and Children feeding on breast milk or Artificial milk formula that contained on DHA in addition to adults from age of 15 t0 25 years. 
- Human milk is the best form of infant nutrition providing significant protection against many illnesses for term and preterm infants.

- This study seeks to investigate the possible protective role of DHA found in Breast Milk or Artificial Milk Formula that are used for feeding of Children and Infants.

- This study would examine the electronic patient record of Newborn from birth to about 2 months of age and Infants from 2 months to 1 year old as well as children from 1year to age 5 years old, feeding on Breast Milk or Artificial Milk Formula between March 2020 and October 2021 and compare their rates of Covid-19 infection, hospitalization and complications with the rates of the local age-matched background population.

- This study will examine the effect of DHA with retinoic acid as a treatment agent in adult patients with COVID-19

- As Covid-19 is a new disease, the existing research literature on this specific topic is extremely limited, and so far this study would be the first in this area.

\section{Declarations}

Ethical Consideration: Before conducting present study, the approval of the ethics committee and the research council of both Kafr-Elshiekh university ,Faculty of Medicine and National committee of Bioethics (H-01-R-009), Riyadh, Saudia Arabia. Written consent will be taken from the study participants (Patients \& control) after explaining the aim of the study to them. All research steps will be recorded in IRCT. Written consent is obtained from all patients before entering the study. We will provide patients with complete and clear information about the research process. At each phase of the study, patients are allowed to leave the study. The medical record of all patients are fully preserved

Conflict of Interest Statement: The author declares that the research was conducted in the absence of any commercial or financial relationships that could be construed as a potential conflict of interest

\section{References}

1-Al-Qahtani AA. Severe Acute Respiratory Syndrome Coronavirus 2 (SARS-CoV-2): Emergence, history, basic and clinical aspects. Saudi J Biol Sci. 2020 Oct;27(10):2531-2538. doi: 10.1016/j.sjbs.2020.04.033. Epub 2020 Apr 23. PMID: 32336927; PMCID: PMC7179492.

2. Lai C.C., Shih T.P., Ko W.C., Tang H.J., Hsueh P.R. Severe acute respiratory syndrome coronavirus 2 (SARS-CoV-2) and corona virus disease-2019 (COVID-19): the epidemic and the challenges. Int $J$ Antimicrob Agents. 2020 Feb 17 doi: 10.1016/j.ijantimicag.2020.105924.

3. Lee PI., Hsueh P.R. Emerging threats from zoonotic coronaviruses-from SARS and MERS to 2019-nCoV. J Microbiol Immunol Infect. 2020;53:365-367. doi: 10.1016/j.jmii.2020.02.001. [ 
4. Wang D., Hu B., Hu C., Zhu F., Liu X., Zhang J.1. Clinical characteristics of 138 hospitalized patients with 2019 novel coronavirus-infected pneumonia in Wuhan, China. J Am Med Assoc. 2020 Feb 7 doi: 10.1001/jama.2020.1585.

5. Huang C., Wang Y., Li X., Ren L., Zhao J., Hu Y. Clinical features of patients infected with 2019 novel coronavirus in Wuhan, China. Lancet. 2020;395:497-506.

6. Li Q., Guan X., Wu P., Wang X., Zhou L., Tong Y. Early transmission dynamics in Wuhan, China, of novel coronavirus-infected pneumonia. N Engl J Med. 2020 doi: 10.1056/NEJMoa2001316. [Epub ahead of print]

7. Ko W.C., Rolain J.M., Lee N.Y., Chen P.L., Huang C.T., Lee P.I. Remdesivir for SARS-CoV-2 pneumonia. Int J Antimicrob Agents. 2020

8. Novel Coronavirus Pneumonia Emergency Response Epidemiology Team . vol. 41. 2020. The epidemiological characteristics of an outbreak of 2019 novel coronavirus diseases (COVID-19) - China, 2020. CCDC weekly 2020;2. Zhonghua Liu Xing Bing Xue Za Zhi; pp. 145-151.

9-Brenna JT, Varamini B, Jensen RG, Diersen-Schade DA, Boettcher JA, Arterburn LM. Docosahexaenoic and arachidonic acid concentrations in human breast milk worldwide. Am J Clin Nutr. 2007 Jun;85(6):1457-64. doi: 10.1093/ajcn/85.6.1457. PMID: 17556680.

10-Lien EL, Richard C, Hoffman DR. DHA and ARA addition to infant formula: Current status and future research directions. Prostaglandins Leukot Essent Fatty Acids. 2018 Jan;128:26-40. doi:

10.1016/j.plefa.2017.09.005. Epub 2017 Sep 15. PMID: 29413359.

11-Gutierrez, S., Svahn, S. L., and Johansson, M. E. (2019). Effects of Omega-3 fatty acids on immune cells. Int. J. Mol. Sci. 20, 5028. doi: 10.3390/ijms20205028

12-Szabó, Zoltán et al. "The Potential Beneficial Effect of EPA and DHA Supplementation Managing Cytokine Storm in Coronavirus Disease." Frontiers in physiology vol. 11752.19 Jun. 2020, doi:10.3389/fphys.2020.00752.

13-https://nutrition.org/the-milk-

debate/\#: :text=Naturally\%2C\%20cow's\%20milk\%20does\%20not,contained\%20in\%20the\%20milk\%20fat

14-Shibabaw T. (2020). Inflammatory Cytokine: IL-17A Signaling Pathway in Patients Present with COVID19 and Current Treatment Strategy. Journal of inflammation research, 13, 673https://doi.org/10.2147/JIR.S278335680.

15-Mousavi Nasl-Khameneh A, Mirshafiey A, Naser Moghadasi A, Chahardoli R, Mahmoudi M, Parastouei K, Yekaninejad MS, Saboor-Yaraghi AA. Combination treatment of docosahexaenoic acid (DHA) and alltrans-retinoic acid (ATRA) inhibit IL-17 and RORyt gene expression in PBMCs of patients with relapsing- 
remitting multiple sclerosis. Neurol Res. 2018 Jan;40(1):11-17. doi: 10.1080/01616412.2017.1382800. Epub 2017 Nov 18. PMID: 29155646.

16-Amr kamel khalil Ahmed, Mahmoud Elkazzaz. Natural phytochemicals, Phenformin, and Docosahexaenoic acid (DHA) as a Novel Inhibitors of IL-6 and ACE2 receptors, a Therapeutic Strategy for targeting COVID-19 Cell Entry and Cytokine Storm. An insilico Approach, 21 September 2021, PREPRINT (Version 1) available at Research Square [https://doi.org/10.21203/rs.3.rs-918251/v1]

\section{Table 1}

Table 1 is available in the Supplementary Files section.

\section{Supplementary Files}

This is a list of supplementary files associated with this preprint. Click to download.

- Table1.png 